\title{
Analysis of Doppler Spread Perturbations in OFDM(A) Systems
}

\author{
Patrick Robertson, Stefan Kaiser \\ Institute for Communications and Navigation, German Aerospace Center (DLR) \\ P.O. Box 1116, D-82230 Wessling, Germany. \\ Tel.: ++498153282808 (2805); FAX: ++49 8153 281442; e-mail: firstname.lastname@dlr.de
}

May 29, 2000

\begin{abstract}
.
We analyze the effects of Doppler spread in mobile channels on Orthogonal Frequency Division Multiplex (OFDM) systems. This is important, since channel variations during one OFDM symbol cause Inter SubCarrier Interference (ICI) in OFDM systems, which degrades the performance, since ICI can be seen as additional near-Gaussian noise. The analysis is presented and closed-form results are given for numerous important practical Doppler spread encountered in mobile channels and applications such as mobile reception of DVB-T. We also show that for the case of asymmetrical Doppler spreads, the frequency correction of the receiver can be adjusted so as to minimize the ICI. To extend our work to OFDM(A) mobile radio systems where many users' contributions to the ICI overlap, we have introduced the concept of an equivalent singleuser which causes the total ICI, in order to use the same analytical framework, and compared the uplink and downlink cases. Simulation results confirm our analysis and show the importance of the analytical tool to perform realistic system analysis without having to resort to time consuming time-domain simulations.
\end{abstract}

\section{Introduction}

In this paper we analyze in depth the perturbations caused by Doppler spreads on multi-carrier systems (in particular OFDM [1, 2]). Basically, the individual carriers in OFDM -when employed in a time invariant multi-path channel- can be made to be orthogonal by the use of a guard interval [1]. However, this orthogonality is destroyed when the channel is time variant, and the channel characteristics change over the duration of one OFDM symbol duration. These changes can be modelled by a Doppler spread: it is the difference in Doppler frequencies affecting different channel paths which lead to the loss of orthogonality, since a common Doppler shift can be viewed as a frequency offset and be corrected [3]. The loss of orthogonality reduces slightly the useful signal in each sub-carrier, and also introduces inter sub-carrier interference (ICI): both effects lead to a reduction in the effective SNR (due to a decrease in the $\mathrm{C} / \mathrm{I}$ ratio) and $\mathrm{C} / \mathrm{I}$ becomes the variable of interest in this work. Unfortunately, most simulations of multi-carrier transmission operate in the frequency domain, and do not model the ICI of the Doppler spread, hence the need for the thorough analysis presented in this paper.

SubAtissamalytical description of the effect of a classical Doppler spectrum on the C/I ratio for OFDM has been introduced in [4] and employed in methods to combat ICI, [5] [6]. A characteristic of the analysis of [4] is the explicit summation over all sub-carriers in the system. We have used a different approach and have found a closed solution for an infinite number of subcarriers -which is still sufficiently accurate for many practical OFDM systems- and classical Doppler distribution, as well as other cases for the Doppler spread, sults. Moreover we have investigated the performance of mobile reception of DVB-T signals [7] for the $2 \mathrm{~K}$ and $8 \mathrm{~K}$ FFT modes.

\section{ICI level in standard OFDM}

'Standard' OFDM here means either point-topoint, or point-to-multi-point transmission; for example broadcasting or the downlink in multi-carrier mobile radio systems. The uplink case has been analyzed in $[8]$.

We begin by presenting a simple model for the OFDM transmission system which is general enough to represent the interference scenario which results from neighbouring sub-carriers cross-talking onto a particular sub-carrier. This so-called inter sub-carrier interference (ICI), is caused by the time variant mobile radio channel which has a significant Doppler spread (in comparison to the sub-carrier spacing).

The base-band model is quite simple and is represented in Fig. 1 with $2 N+1$ sub-carriers. The pulseshaping filters $g(t)$ and $h(t)$ are matched, and we assume rectangular (in time) forms for both, where $T$ is the useful OFDM symbol length. After the matched filter, we have the sampler for each sub-carrier, which would be followed by, e.g. the decision unit. We are interested in the levels of ICI and useful signal after the decision unit. The multi-path channel model is drawn in Fig. 2. The channel models $P$ paths, each with index $k$, a Doppler frequency $f_{d}^{(k)}$, attenuation $e^{j \alpha^{(k)}} \cdot a^{(k)}$, and delay $\tau^{(k)}$.

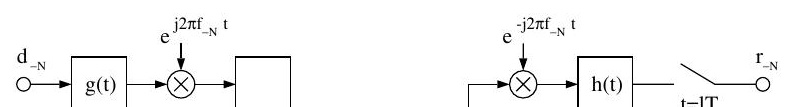




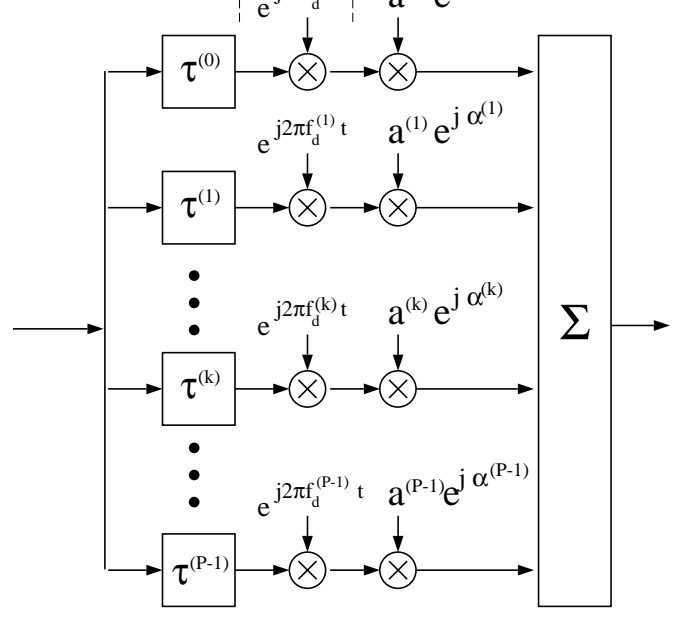

Figure 2: The channel model with $P$ paths, each with a Doppler frequency, attenuation and delay.

ing from the Doppler spread. We have not in principle excluded the extension where we look at a useful subcarrier that lies near the edge of the OFDM spectrum (e.g. at index $N$ or $N-1$ ), since these sub-carriers will suffer from less ICI.

\subsection{ICI from one disturbing sub carrier}

Fortunately, the delay of each path is irrelevant to our analysis if all echos lie within the cyclic extension employed in OFDM. Observe that the ICI is caused by different paths having different Doppler frequencies, which break the orthogonality between the sub-carriers. Different echo delays $\tau^{(k)}$ and different phases $\alpha^{(k)}$ will essentially result in different overall phase rotations in the amplitude "leakage" from any sub-carrier $i, i \neq 0$, onto our useful sub-carrier with index 0 , as far as the contribution via the channel path $k$ is concerned.

The individual ICI components which will additively contribute to the total ICI are obtained through varying all sub-carriers with index $i$ and all paths with their index $k$. Because of statistically independent phases for each path, and statistically independent data $d_{i}$ on each sub-carrier $i$, all these individual noise components are uncorrelated, which is easy to prove. Therefore, we can sum their energies when computing the ICI energy and are able to ignore the phase rotation of each path, and, of course, the actual phase values of the data symbols transmitted on each subcarrier. Moreover, the components are statistically independent when the channel paths' Doppler frequencies and amplitudes are constant; since this is the case for at least the duration of one OFDM symbol, we can use the central limit theorem for sufficiently large $N$ to model the ICI as zero-mean additive Gaussian noise. a)

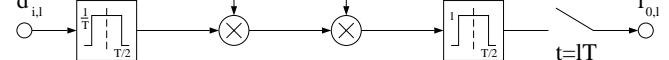

b)

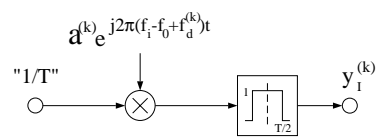

Figure 3: The simplified transmission model: a) assuming only one disturbing sub-carrier $i$ and observing received sub-carrier 0 , there is one echo, $k$. b) Setting $d_{i, l}=1 \forall l$ allows one to ignore the sampling when determining the variance of the samples $r_{0, l}^{(k)}$.

To proceed, let us simplify the model further. The top half of Fig. 3 shows the data symbol of sub-carrier $i$ for OFDM symbol $l$, and how it influences the subcarrier zero, for one echo only, namely with index $k$. Furthermore, the phases of the channel paths have been set to zero, which we may do for reasons of symmetry. The ICI power in both I and Q components will in reality be the same, but by setting the phases to zero we have forced all the ICI to affect the I component only, whose energy will now be equal to the sum of the ICI energy in the I and Q components in the real system. Thus our analysis pertains to the total ICI energy in I and Q. In order to continue, the model in the top half of Fig. 3 must again be simplified. To determine the variance of the samples $r_{0, l}^{(k)}$ (for a given energy of $d_{i, l}$ ), the sampling can be ignored if we are able to set $d_{i, l}=1, \forall l$. This restriction can be justified since the system is linear and the data of one OFDM symbol $l$ will not affect the output of the receiver for other OFDM symbols $\neq l$. In this case the variance of $y_{I}^{(k)}$ will equal that of $r_{0, l}^{(k)}$ as long as $E\left\{d_{i, l}^{2}\right\}=1$. This leads to the simple model of Fig. $3 \mathrm{~b}$ ), where we now need to calculate the variance of $y_{I}^{(k)}$, which is the integral

$$
\begin{gathered}
\sigma_{y_{I}^{(k)}}^{2}=\int_{-\infty}^{\infty}\left(\frac{1}{T} \cdot a^{(k)}\right)^{2} \delta\left(f-\left(f_{i}-f_{0}+f_{d}^{(k)}\right)\right) \\
\cdot T^{2} \cdot \operatorname{sinc}^{2}(\pi f T) d f
\end{gathered}
$$

since the spectrum of the signal at the input of the rectangular filter is a Dirac delta with amplitude $\frac{1}{T}$. $a^{(k)}$, at frequency $f=f_{i}-f_{0}+f_{d}^{(k)}$, and the power density spectrum (PDS) of the filter is $T^{2} \cdot \operatorname{sinc}^{2}(\pi f T)$. Thus the variance is:

$$
\sigma_{y_{I}^{(k)}}^{2}=\left(a^{(k)}\right)^{2} \operatorname{sinc}^{2}\left(\pi\left(f_{i}-f_{0}+f_{d}^{(k)}\right) T\right) .
$$


The useful signal energy $N_{u}^{(k)}$ which is contributed by one path with Doppler frequency $f_{d}^{(k)}$ is easily computed by setting $i=0$ in (2), yielding $N_{u}^{(k)}$ $=\left(a^{(k)}\right)^{2} \operatorname{sinc}^{2}\left(\pi\left(f_{d}^{(k)}\right) T\right)$. Since all paths have independent phases, and hence all useful signal components are uncorrelated, we are able to compute the total useful energy as the sum of $N_{u}^{(k)}$ over all $k$.

2.3 Summation of the ICI over a finite number of neighbouring sub-carriers

Let $L_{t}(k)$ denote the total ICI energy which results from all sub-carriers onto the sub-carrier with index 0 , as a function of the Doppler frequency $f_{d}^{(k)}$, for a single path with index $k$. To compute $L_{t}(k)$, we sum all the $\sigma_{y_{I}^{(k)}}^{2}$ :

$$
\begin{gathered}
L_{t}(k)=\sum_{i=-N}^{-1}\left(a^{(k)}\right)^{2} \operatorname{sinc}^{2}\left(\pi\left(f_{i}-f_{0}+f_{d}^{(k)}\right) T\right)+ \\
\sum_{i=1}^{N}\left(a^{(k)}\right)^{2} \operatorname{sinc}^{2}\left(\pi\left(f_{i}-f_{0}+f_{d}^{(k)}\right) T\right) .
\end{gathered}
$$

Normalizing the symbol duration $T$ to unity for simplicity, evaluation of these sums yields,

$$
\begin{aligned}
& L_{t}(k)=\left(a^{(k)} \cdot \operatorname{sinc}\left(\pi f_{d}^{(k)}\right)\right)^{2} . \\
& \left(\left(f_{d}^{(k)}\right)^{2} \cdot\left(\psi^{\prime}\left(-f_{d}^{(k)}-N\right)-\psi^{\prime}\left(1-f_{d}^{(k)}+N\right)\right)-1\right),
\end{aligned}
$$

where $\psi^{\prime}(x)$ is the first derivative of the digamma function [9]. We have used the name "leakage" function for $L_{t}$, since it represents the sum of the energy that leaks over from all carriers onto our chosen target carrier with index zero, due to a particular channel path $k$ with a particular Doppler frequency $f_{d}^{(k)}$.

2.4 Summation of the ICI over a infinite number of neighbouring sub-carriers

The easier case is if we let $N$ go to infinity, i.e. using (3) we evaluate $\lim _{N \rightarrow \infty} L_{t}(k)$. The result can be computed straightforwardly to be (again after normalizing the symbol duration $T$ to unity):

$$
L_{t}(k)=\left(a^{(k)}\right)^{2} \cdot\left[1-\operatorname{sinc}^{2}\left(\pi f_{d}^{(k)}\right)\right] .
$$

In practice, we have observed that using the easier case of infinite sub-carriers yields virtually the same result as for finite carriers, for 60 or more carriers in the OFDM system. Using (5) makes the following analysis much easier.

So far, the leakage function $L_{t}(k)$, expressed in equations (4) and (5), holds for one path with index $k$, Doppler frequency $f_{d}^{(k)}$, and amplitude $a^{(k)}$. The total ICI energy experienced by carrier with index zero is thus just the sum $N_{t}=\sum_{\forall k} L_{t}(k)$. Now note that because $L_{t}(k)$ is proportional to $\left(a^{(k)}\right)^{2}$, we can combine all those paths with exactly the same Doppler frequency, and add their energies $\left(a^{(k)}\right)^{2}$, to make up a new, hypothetical path of greater energy $\left(a_{f_{d}}\right)^{2}=$ $a^{2} \cdot p\left(f_{d}\right)$, which contributes $L_{t}\left(f_{d}\right)$. The new variable $a^{2}$ is the sum of the energy of all paths of the channel (i.e. summed over all Doppler frequencies). It is formally needed because the integral over $p\left(f_{d}\right)$ has to be unity, but not the integral over $a^{2} \cdot p\left(f_{d}\right)$. Therefore, if we are given a statistical distribution for the Doppler frequency, $p\left(f_{d}\right)$, the expression for $N_{t}$ can be written as:

$$
N_{t}=a^{2} \cdot \int_{-\infty}^{\infty} L_{t}\left(f_{d}\right) \cdot p\left(f_{d}\right) \cdot d f_{d}
$$

The product $a^{2} \cdot p\left(f_{d}\right)$ will be denoted in this paper as the weighted PDF; unless otherwise stated it will in fact equal $p\left(f_{d}\right)$.

\subsubsection{Definition of Doppler Frequency PDFs}

Discrete: Physically, this distribution of $f_{d}$ corresponds to a finite number of paths, each with a given Doppler frequency. Note that we do not have to separate physical paths with different delays, but the same Doppler frequency. We denote the number of such distinct paths with $P$, and use index $k^{\prime}$ in order to avoid confusion with $k$, which might be different if some physical paths have the same Doppler frequency.

A special case is a two-path model, which is both simple to compute, and will lend itself to the derivation of the optimal frequency to which the receiver should adjust, in order to minimize the ICI, see Section 3. Furthermore, it shall serve as an approximation to a classical Doppler spectrum. It is modeled with $a=1$, and the following PDF for the Doppler frequency:

$$
p\left(f_{d}\right)=\left(1-p_{2}\right) \delta\left(f_{d}-f_{1}\right)+p_{2} \delta\left(f_{d}-\left(f_{1}+\Delta f\right)\right)
$$

The variable $f_{1}$ denotes the Doppler frequency of one of the paths and $p_{2}$ the energy of the other path. The frequency distance between the two paths is given by $\Delta f$.

Uniform: It is useful to consider a uniform distribution, with maximal Doppler frequencies $f_{d_{\max }}$, as it turns out to be easy to handle analytically, and, moreover, it corresponds to a number of physically interesting cases. Notably, it has been shown in [10], that for scattering environments in three dimensions the PDF 
For a uniform distribution, we have found the fol-

Classical: The classical Doppler spectrum results from uniformly distributed angels of arrival at the receiver antenna. The PDF for the classical Doppler case is $[11]$

$$
p\left(f_{d}\right)=\frac{1}{\pi \cdot f_{d_{\max }} \cdot \sqrt{1-\left(\frac{f_{d}}{f_{d_{\max }}}\right)^{2}}} ; \text { for }\left|f_{d}\right| \leq f_{d_{\max }} .
$$

The classical Doppler spectrum is a long term average. For considerable lengths of time we will often observe an asymmetrical or biased Doppler spectrum, which we approximate through our discrete two-path model above, with $p_{2} \neq 0.5$.

Gaussian with Zero Mean: The PDF is,

$$
p\left(f_{d}\right)=\frac{1}{\sigma_{f_{d}} \sqrt{2 \pi}} \cdot e^{-\left(\frac{f_{d}}{2 \sigma_{f_{d}}}\right)^{2}}
$$

where $\sigma_{f_{d}}$ is the standard deviation of $f_{d}$.

\subsection{Integration for Finite and Infinite Sub-Carriers}

We are now able to proceed with the integration to evaluate (6). The integration is straightforward and leads to closed-form solutions for an infinite number of carriers (albeit resorting to the use of special functions in the solutions).

\subsubsection{Discrete}

To be consistent with the cases of continuous PDFs, we shall evaluate (6), where $E^{\left(k^{\prime}\right)}$ is the energy of all paths with a distinct Doppler frequency $f_{d}$ $=f_{d}^{\left(k^{\prime}\right)}$. Of course, equations (4) and (5) are formally computed by simply setting $a^{(k)}$ to unity, and $f_{d}^{(k)}$ to $f_{d}^{\left(k^{\prime}\right)}$. This yields,

$$
\begin{gathered}
N_{t}=\sum_{k^{\prime}=0}^{P-1} L_{t}\left(f_{d}^{\left(k^{\prime}\right)}\right) \cdot E^{\left(k^{\prime}\right)} . \\
P_{u}=\sum_{k^{\prime}=0}^{P-1} \operatorname{sinc}^{2}\left(\pi\left(f_{d}^{\left(k^{\prime}\right)}\right) T\right) \cdot E^{\left(k^{\prime}\right)} .
\end{gathered}
$$

For the two-path model the useful signal energy is:

$$
P_{u}=\left(1-p_{2}\right) \operatorname{sinc}^{2}\left(f_{1} \pi\right)+p_{2} \operatorname{sinc}^{2}\left(\Delta f+f_{1} \pi\right) .
$$

For an infinite number of sub-carriers, the ICI energy

$$
\begin{aligned}
& \quad N_{t}= \\
& \left(1-p_{2}\right)\left[1-\operatorname{sinc}^{2}\left(f_{1} \pi\right)\right]+p_{2}\left[1-\operatorname{sinc}^{2}\left(\Delta f+f_{1} \pi\right)\right] .
\end{aligned}
$$

lowing solution for an infinite number of carriers,

$$
\begin{aligned}
N_{t}= & \frac{\cos \left(2 \pi f_{d_{\max }}\right)+2 f_{d_{\max }} \pi \operatorname{Si}\left(2 f_{d_{\max }} \pi\right)}{2 \cdot\left(\pi \cdot f_{d_{\max }}\right)^{2}}+ \\
& \frac{-1-2 \cdot\left(\pi \cdot f_{d_{\max }}\right)^{2}}{2 \cdot\left(\pi \cdot f_{d_{\max }}\right)^{2}}
\end{aligned}
$$

where $\operatorname{Si}($.$) is the sine integral function [9]. Unfortu-$ nately, we have had to resort to numerical integration for the finite sub-carrier situation.

The useful energy is always

$$
P_{u}=-\frac{\cos \left(2 \pi f_{d_{\max }}\right)+2 f_{d_{\max }} \pi \operatorname{Si}\left(2 f_{d_{\max }} \pi\right)-1}{2\left(\pi \cdot f_{d_{\max }}\right)^{2}} .
$$

\subsubsection{Classical}

For infinite sub-carriers, we have used the relation $\sin ^{2}(x)=\frac{1}{2}(1-\cos (2 x))$, and inserted this into the integral,

$$
I_{c d}=\int_{-f_{d_{\max }}}^{f_{d_{\max }}} \frac{1-\operatorname{sinc}^{2}\left(f_{d} \pi\right)}{f_{d_{\max }} \cdot \pi \cdot \sqrt{1-\left(\frac{f_{d}}{f_{d_{\max }}}\right)^{2}}} d f_{d} .
$$

Using [12] to continue, we can express the solution using the generalized hyper-geometric function [9], ${ }_{p} \mathrm{~F}_{q}$, where in our case, $p=1, q=2$ :

$$
N_{t}=1-{ }_{1} \mathrm{~F}_{2}\left(\frac{1}{2} ; \frac{3}{2}, 2 ;-\left(f_{d_{\max }} \pi\right)^{2}\right) \text {. }
$$

For finite sub-carriers, we again employ numerical integration.

In all cases, the useful energy is

$$
P_{u}={ }_{1} \mathrm{~F}_{2}\left(\frac{1}{2} ; \frac{3}{2}, 2 ;-\left(f_{d_{\max }} \pi\right)^{2}\right)
$$

\subsubsection{Gaussian}

For a Gaussian distribution with zero mean and infinite sub-carriers, the final result is

$$
\begin{aligned}
& N_{t}=
\end{aligned}
$$

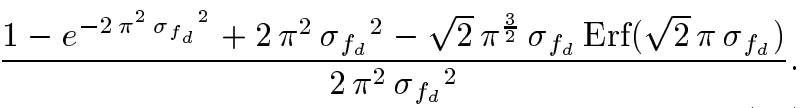

The useful energy is

$$
P_{u}=\frac{-1+e^{-2 \pi^{2}{\sigma_{f_{d}}}^{2}}+\sqrt{2} \pi^{\frac{3}{2}} \sigma_{f_{d}} \operatorname{Erf}\left(\sqrt{2} \pi \sigma_{f_{d}}\right)}{2 \pi^{2}{\sigma_{f_{d}}}^{2}} .
$$




\subsection{Solution for small Doppler Spreads}

For small $f_{d}$, infinite sub-carriers, and an otherwise arbitrary PDF, the minimal ICI is achieved when the expected value of the Doppler frequency is zero at the receiver, since for small values of $x$, we can use the approximation $1-\operatorname{sinc}^{2}(x) \approx \frac{1}{3} \cdot x^{2}$ in $(5)$ and in the expression for $N_{u}^{(k)}$, leading to a quadratic relation between $L_{t}(k)$ (or $N_{u}^{(k)}$ ) and $f_{d}$. In other words, a frequency synchronization algorithm which sets the mean Doppler frequency to zero will be close-to-optimal with respect to ICI minimization as long as the Doppler spread is small. For the two path model (7), it is very easy to show that this yields a much easier approximately optimal solution for $f_{1}$, namely $f_{1} \approx-\Delta f \cdot p_{2}$.

\subsection{Solution for large Doppler Spreads}

We will restrict ourselves to the two-path model with weighted Doppler PDF (7). We shall let $f_{1}$ be the free variable, and observe the variation of the $\mathrm{C} / \mathrm{I}$ ratio. When we find the value of $f_{1}$ which maximizes this ratio, we have found the best frequency for the receiver's local oscillator, namely the one which results in the value of $f_{1}$ for the path in the weighted Doppler PDF with energy $\left(1-p_{2}\right)$. The values of $p_{2}$ and $\Delta f$ are fixed for one determination of the best $f_{1}$.

To do this, we differentiate either the useful signal energy (12) or the ICI energy (13) with respect to $f_{1}$ : notice that the result is the same except for the sign. Setting this derivative to zero and solving the resulting equation to find $f_{1}$ involves either a numerical solution, or a Taylor series expansion followed by an approximate analytical solution of the resulting truncated series, set to zero. We have opted for a second order expansion, where we have developed the Taylor series around the value of $f_{1}=-\Delta f \cdot p_{2}$, using [12]. The resulting analytical expression for $f_{1}$ yields two solutions, which both have to be inserted into (13), together with $\Delta f$ and $p_{2}$, to find the one yielding the lowest value of the ICI. The equation has been omitted here for brevity.

\subsubsection{Performance Gain}

We shall now compare the gain achieved by using the optimal solution for $f_{1}$, compared to the approximation $f_{1} \approx-\Delta f \cdot p_{2}$. We have shown the gain in $\mathrm{dB}$ for different values of $p_{2}$ and $\Delta f \cdot T$ in Fig. 4 . As can be seen, there is little to be gained for values of $\Delta f \cdot T$ less than 0.5. However, for values greater than 0.5 , the gain can be significant even for small values of $p_{2}$. This implies that even weak components at high Doppler frequencies (relative to the most powerful path) can the receiver synchronisation design. It is important

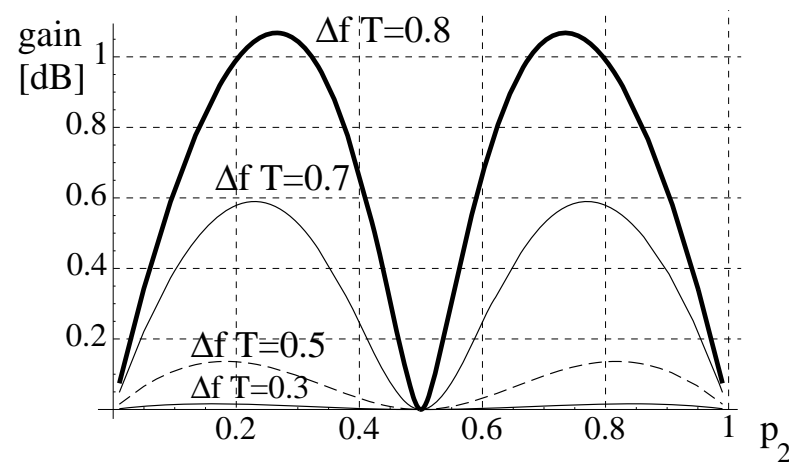

Figure 4: The gain from using the optimal frequency correction for different $\Delta f \cdot T$ and $p_{2}$ for a two path model.

to remember that these results only apply to the very simple two path model.

It appears that a frequency synchronizer which minimizes the ICI directly (for example by observing the $\mathrm{C} / \mathrm{I}$ of some sub-carriers and maximizing), can provide some gain compared to one which sets the average Doppler frequency to zero. Furthermore, such a synchronizer will per definition find the optimal frequency without having to know the solution for each model for the distribution of the Doppler frequencies.

\section{Uplink Case for $\operatorname{OFDM}(\mathrm{A})$}

In a model for the uplink of mobile radio $\operatorname{OFDM}(\mathrm{A})$ schemes or MC-FDMA schemes [13], we distinguish two cases: 1) Each mobile user transmits with its nominal carrier frequency; it does not adapt this to its Doppler spread experienced in the downlink, which might be unrelated to the uplink. Also, the basestation receiver does not change its local oscillator frequency for each detected user. This case corresponds to the more simple situation in which the mobile does not necessarily have access to a downlink, or where the downlink suffers from a different Doppler spread (frequency division duplex (FDD), e.g.). The resulting equivalent Doppler frequency PDF is that which is obtained when all users are merged to a single, hypothetical, user. Case 2): Each mobile transmits with a corrected frequency, depending on the Doppler spread it measures in the downlink: Time division duplex (TDD) is assumed, or a downlink indicating the necessary frequency shift value for the uplink of each user. This is the more suitable case, giving improvements if the short term Doppler spectra of some of the users are asymmetrical. The base station receiver has no need to change the frequency for each user it detects. The in- 
the channel for a user is assumed to be the same in the uplink and the downlink, and the ICI in the uplink is the sum of all users' ICI contributions, the total uplink ICI is also minimized. For a discrete two-path model and four disturbing users we have given an example in Fig. 5 for these two cases. For case 2), the two powerful paths have a lower Doppler frequency due to the frequency correction at each mobile's transmitter, resulting in lower ICI. In Fig. 6 the simulated equiva-

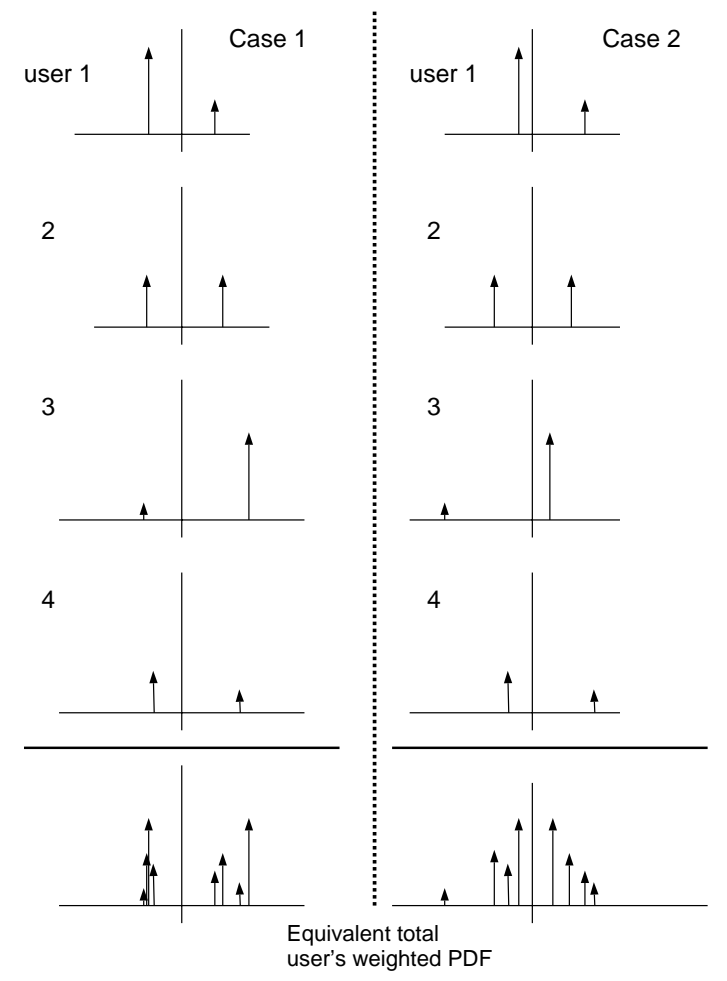

Figure 5: The resulting weighted PDF of the equivalent user for cases 1) and 2). There are 4 uplink users, each of these has a different (shown) two path channel.

lent user's PDF according to case 1) without frequency correction and to case 2) with frequency are plotted. It can be observed that with frequency correction the power is located more in the center, resulting in less ICI.

\section{Results}

The parameters of the analyzed OFDM system (for a mobile radio scenario) are as follows: The bandwidth is $B=2 \mathrm{MHz}$ and the carrier frequency is located at $f_{c}=2 \mathrm{GHz}$. The number of sub-carries for OFDM is $N=256$. Thus, the carrier distance is $7.81 \mathrm{kHz}$. We have chosen QPSK for symbol mapping. The maximum number of active users (fully loaded

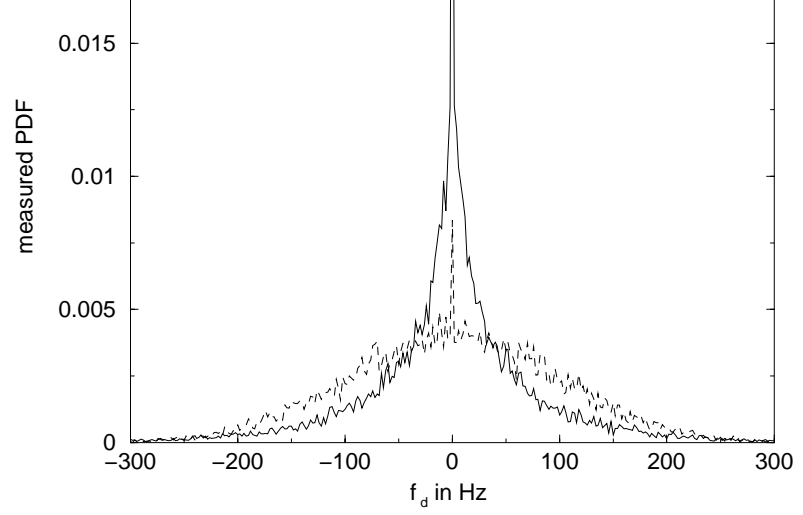

Figure 6: Simulated PDF in the uplink.

system) is 32 , so each user transmits exclusively on its 8 sub-carriers. This principle is called MC-FDMA or OFDMA [13]. The 8 sub-carriers of a single user are block interleaved such that they have maximum frequency distance. The data rate per user is $125 \mathrm{kbit} / \mathrm{s}$.

The $\mathrm{C} / \mathrm{I}$ is evaluated separately for the downlink and the uplink. For the former, we have defined 6 cases with different assumptions about the multi-path propagation in the channel: 1) classical Doppler spectrum 2) 2-path, symmetrical PDF, 3) 2-path, asymmetrical PDF, $20 \%$ and $80 \%$ energy distribution, 4) 2-path, asymmetrical PDF, $10 \%$ and 90\%, 5) 2-path, asymmetrical PDF, $30 \%$ and $70 \%, 6)$ uniform Doppler spectrum.

For the uplink we defined 2 cases based on a two path model with different assumptions on the Doppler spectra: The maximum Doppler frequencies $f_{d_{\max }, \text { user }}$ of the individual users is taken in case a) as the absolute value of a zero-mean Gaussian RV with $\sigma=$ $0.5 \cdot f_{d_{\text {max }}}$, and in case b) from a uniform distribution in $\left(0, f_{d_{\max }}\right)$. The power of $p_{2}$ is taken from a uniform distribution in $[0,1]$ in both cases.

The $\mathrm{C} / \mathrm{I}$ versus the Doppler frequency is shown in Fig. 7 for the downlink. Analytical and simulation results are shown without frequency correction. Additionally, results are presented with optimal frequency correction according to Section 3 for those cases where it is applicable (asymmetric PDFs). As is evident from the figure, the analytical results match perfectly with the simulation results. The $\mathrm{C} / \mathrm{I}$ versus the Doppler frequency is shown in Fig. 8 for the uplink, and was only evaluated by simulations. The $\mathrm{C} / \mathrm{I}$ is plotted for the case with and without frequency correction.

Finally, we have shown analytical results in Fig. 9 for the DVB-T terrestrial broadcasting system [7] and its mobile reception for different channels and for the $2 \mathrm{~K}$ and $8 \mathrm{~K}$ FFT modes. The assumed carrier was at $800 \mathrm{MHz}$ and the $C / I$ ratio is plotted against the vehicle velocity. The performance of the $8 \mathrm{~K}$ mode seems 
fourier transform," IEEE Trans. Commun., vol. 19, pp. 628-634, October 1971.

[3] P. H. Moose, "A technique for orthogonal frequency division multiplexing frequency offset correction," IEEE Trans. Commun., vol. 42, pp. 2908-2914, October 1994.

[4] M. Russel and G. Stüber, "Terrestrial digital video broadcasting for mobile reception using OFDM," Wireless Personal Comms, Special Issue Multi-Carrier Comms, vol. 2, no. 1\&2, pp. 45-66, 1995.

[5] K. Matheus and K.-D. Kammeyer, "Optimal design of multicarrier systems with soft impulse shaping including equalization in time or frequency," in Proc. GLOBECOM '97, pp. 310-314, 1997.

[6] J. Armstrong, P. Grant, and G. Porey, "Polynomial cancellation of OFDM to reduce intercarrier interference due to doppler spread," in Proc. GLOBECOM '98, 1998.

[7] DVB-T, "Digital broadcasting systems for television, sound, and data services; frame structure, channel coding and modulation for digital terrestrial broadcasting," No. ETS 300 744, (Standard), 1996.

[8] P. Robertson and S. Kaiser, "The effects of Doppler spreads in OFDM(A) mobile radio systems," pp. 329333, September 1999. Proc. VTC'99.

[9] I. S. Gradshteyn and I. Ryzhik, Tables of Integrals, Series and Products. Academic Press, 1965.

[10] "Final report on RF channel characterization." JTC (AIR) 93.09.23-238R2, 1993.

[11] W. C. Jakes, Microwave Mobile Communications. John Wiley \& Sons, Inc., 1974.

[12] S. Wolfram, The Mathematica Book, 3rd ed. Wolfram Media, Cambridge Univ. Press, 1996.

[13] S. Kaiser, "MC-FDMA and MC-TDMA versus MCCDMA and SS-MC-MA: Performace evaluation for fading channels," in Proc. IEEE Fifth Int. Symp. on Spread Spectrum Techniques \& Applications (ISSSTA'98), pp. 200-204, Sept. 1998. 